\title{
Prevalence of Bovine Tuberculosis Using Abattoir Meat Inspection in Cattle Slaughtered at Gondar Elfora Abattoir, North Ethiopia
}

\author{
Tarekegn Tintagu Gizaw ${ }^{*}$, Bethelehem Alemu Minalu, Yalew Tefera Asfaw
}

School of Veterinary Medicine, Wollo University, Dessie, Ethiopia

Email address:

drtarekegn@yahoo.com (T. T. Gizaw)

${ }^{*}$ Corresponding author

\section{To cite this article:}

Tarekegn Tintagu Gizaw, Bethelehem Alemu Minalu, Yalew Tefera Asfaw. Prevalence of Bovine Tuberculosis Using Abattoir Meat Inspection in Cattle Slaughtered at Gondar Elfora Abattoir, North Ethiopia. Animal and Veterinary Sciences. Vol. 5, No. 5, 2017, pp. 89-96. doi: $10.11648 /$ j.avs.20170505.15

Received: September 16, 2017; Accepted: September 28, 2017; Published: October 30, 2017

\begin{abstract}
Bovine tuberculosis (BTB) is endemic in Ethiopia and like other developing countries in Africa. Ethiopia has limited laboratory and other diagnostic facilities. Therefore, abattoir inspection still remains the only option for monitoring BTB prevalence in domestic animals. A cross-sectional type of study was conducted at Gondar ELFORA abattoir from October, 2009 to April, 2010 to assess the prevalence of BTB slaughtered cattle. During this time, a total of 500 animals under gone on ante mortem and detailed post mortem inspections and $43(8.6 \%)$ of the animals were found to be with tuberculous lesions. About $84.8 \%$ of the tuberculous lesions were found in the lymph nodes of the thoracic cavity (mediastinal and bronchial), followed by $11.6 \%$ in the lymph nodes of the abdominal cavity (mesenteric) and the remaining $4.65 \%$ in the lymph nodes of the head (retropharyngeal). From the 43 cattle samples that were suspicious to contain tuberculous lesions, samples of lymph nodes were cultured on Lowenstein-Jensen media and growth of Mycobacterium was observed on 5/43(11.6\%) samples. In this study, age $\left(\chi^{2}=3.351, \mathrm{p}\right.$ value $\left.=0.187\right)$ and breed $\left(\chi^{2}=1.087, \mathrm{p}\right.$ value $\left.=0.29\right)$ have not shown any association with post mortem results whereas origin $\left(\chi^{2}=147.179, p\right.$ value $\left.=0.0252\right)$, anatomical sites $\left(\chi^{2}=468.814, p\right.$ value $\left.=0.00\right)$, sex $\left(\chi^{2}=23.688, p\right.$ value $=0.011)$, body condition score $\left(\chi^{2}=17.342, p\right.$ value $\left.=0.00\right)$ and culture results $\left(\chi^{2}=121.48, p\right.$ value $\left.=0.00\right)$ have shown associations with post mortem result. Based on this study, it is recommended that integrated preventive approaches involving enhanced surveillance of the disease through establishment of standardized abattoirs well-equipped with laboratory facilities.
\end{abstract}

Keywords: Bovine, Cross-Sectional, Culture, M. Bovis, Risk Factors

\section{Introduction}

Tuberculosis (TB) is one of the leading causes of infectious disease mortality in the world, with over 2 million deaths recorded annually, and it is estimated that one third of the world's population is latently infected. Together with HIV or AIDS and Malaria, tuberculosis (TB) is recognized as one of the most important threats to human health. There are around 9 million new cases of TB every year, resulting in up to 2 million deaths. Tuberculosis (TB) is an infectious disease caused by Mycobacteria that have been a major health risk to humans and animals for more than a century. It is widely distributed throughout the world affecting all age groups of humans and animals [1].
Despite intensive efforts over decades, bovine tuberculosis (BTB) continues to be significant global problem [2]. The impact of the disease varies both by continent and economic status of individual countries. In countries where BTB is still common and pasteurization of milk is not practiced, an estimated 10-15\% of human TB is caused by Mycobacterium bovis [3]. However, in all regions where it is present it has an adverse influence on agricultural economics [2] particularly on the international trade of animals and animal products [4].

Most Mycobacteria are classified into two categories: fastgrowing and slow-growing, based on laboratory growth characteristics. All Mycobacteria are aerobic, acid fast and share a characteristic cell wall, thicker than in many other bacteria, which is hydrophobic, waxy and rich in mycolic 
acids/mycolates and the Mycobacterial cell wall makes a substantial contribution to the hardiness of this genus. Mycobacteria tend to be difficult to culture, as well as being fastidious in growth. M. bovis belongs to a group of closely related organisms known as the Mycobacterium tuberculosis complex (MTBC) that comprises seven recognized members M. tuberculosis, M. bovis, M. africanum, M. pinnipedii $M$. caprae, M. microti and M. Canetti [1].

The signs vary with the distribution of tubercles in the body but, with few exceptions, the course of the disease is chronic. In many instances, characteristic signs are lacking, even in advanced stages of the disease when many organs may be involved. Lung involvement may be manifested by a cough, which can be induced by changes in temperature or manual pressure on the trachea. Dyspnea and other signs of low-grade pneumonia are also evidence of lung involvement. In advanced cases, lymph nodes are often greatly enlarged and may obstruct air passages, the alimentary tract or blood vessels. Lymph nodes of the head and neck may become visibly affected and sometimes rupture and drain. Extreme emaciation and acute respiratory distress may occur during the terminal stages of tuberculosis. Tubercles of cattle are most frequently seen at necropsy in bronchial, mediastinal, retropharyngeal and portal lymph nodes and may be the only tissue affected. In addition, the lung, liver, spleen and the surfaces of body cavities are commonly affected. Other anatomical sites must be considered as potential to become infected. At necropsy, a tuberculous granuloma usually has a yellowish appearance and is caseous, caseo-calcareous, or calcified in consistency. The caseous center is usually dry, firm, and covered with a fibrous connective capsule of varying thickness $[1,5]$.

Despite the ability of several countries to eradicate bovine tuberculosis successfully, many countries continue to encounter M. bovis infection in their cattle population [6]. Reasons for the failure of these countries to control/eradicate the disease include the presence of feral reservoirs of $M$. bovis in developed countries to apply the test and slaughter control method. Other alternative control methods such as the use of vaccination, improvement of cattle husbandry and/or breeding of relatively resistant cattle breeds may be particularly applicable in developing countries. Zebu type cattle are thought to be more resistant to tuberculosis than European cattle and the effects of the disease on these cattle are much less severe as stated earlier [7].

In industrialized countries, BTB is controlled by testing and slaughter of animals and pasteurization of milk and therefore the risk of human infection are minimized. Although effective drugs are available for most cases and rates of $\mathrm{TB}$ infection have decreased dramatically in industrialized countries poor public health systems in developing countries, the HIV/AIDS pandemic and the emergence of multidrug-resistant strains, have all contributed to an ongoing increase in reported cases worldwide. In Africa, however, bovine TB represents potential health hazards for both animals and humans. Nonetheless, in most African countries, M. bovis infection remains an uninvestigated problem [7].

Ethiopia is a country where the impact of BTB is particularly important and it is amongst the three African countries with the highest burden of human TB cases [6]. Similarly, the prevalence of BTB in Ethiopia is also high, ranging from $3.4 \%$ in smallholder production systems that keep Zebu cattle to $50 \%$ in peri-urban (intensive) dairy production systems $[8,11]$. The high prevalence of TB in cattle, the close contact of cattle and humans in rural areas, the habit within the community of consuming raw milk and the increasing prevalence of HIV may all increase the potential for transmission of $M$. tuberculosis and other Mycobacteria between cattle and their owners [12].

Diagnosis of $M$. bovis infection in cattle is often based on history, clinical and necropsy findings and tuberculin test. Necropsy procedure represents the most acceptable means of detecting tuberculous lesions in cattle [12]. However, detection of tuberculosis by slaughter surveillance required that infected cattle have visible lesions at inspected sites but not all infected cattle develop visible lesions in sites that are examined [13]. In abattoir, smaller lesions may have been missed because there is limited time available for examination of each tissue and fine slicing the lymph nodes in situ is very difficult. Furthermore, routine meat inspection can lead to the classification of non-tuberculous lesions as tuberculous due to the similarity in macroscopic appearance [14].

Several laboratory procedures have been introduced as aids in the diagnosis of Mycobacterial infections. However, due to technical problems and cost, they have not come into wide used in veterinary diagnostic laboratories [15]. A tentative diagnosis of BTB can be made following the finding of typical lesions at necropsy examinations. These lesions should be collected for laboratory confirmation of $M$. bovis infection. Though presumptive diagnosis can be made using histopathology, a definitive diagnosis of $M$. bovis infection often requires isolation and identification of the causative agent from tissue specimens, morphological appearance and biochemical and drug sensitivity tests [16]. In vitro lymphocyte stimulation assay, interferon gamma assay and ELISA can also be used for the diagnosis of BTB at various levels. To confirm the diagnosis, culture and biochemical properties are used. To differentiate M. bovis from other members of Mycobacterium tuberculosis complex, molecular techniques based on polymerase chain reaction (PCR) and Deoxyribonucleic acid (DNA) genome sequence are used in laboratories where the facilities are available [15].

In countries like Ethiopia, implementation of detailed necropsy examination of carcasses at slaughter houses continues to be an important step in surveillance [17]. The present study was conducted on cattle slaughtered at Gondar ELFORA abattoir; a number of people are involved in feed lot production systems supplying cattle to surrounding slaughter house. A more serious focus on bovine TB status as well as accurate estimation of the magnitude and distribution of bovine $\mathrm{TB}$ in cattle are essential for appropriate 
intervention strategies. This study was carried out to ascertain the magnitude and trend of detection of TB lesions in slaughtered cattle at Gondar ELFORA abattoir. Therefore, the objectives of this study were; to assess the prevalence of BTB slaughtered cattle in Gondar ELFORA abattoir, to determine the importance of anatomical sites involved in harboring tuberculous lesions at post mortem examinations and to isolate of $M$. bovis from suspected tuberculous lesions via culture techniques

\section{Materials and Methods}

\subsection{Study Area}

Gondar is a city located in the North Gondar Zone of the Amhara Region, Gondar is north of Tana Lake on the Lesser Angereb River and southwest of the Simien Mountains. It has a latitude and longitude of $12^{\circ} 36^{\prime} \mathrm{N} 37^{\circ} 28^{\prime} \mathrm{E}$ with an elevation of 2133 meters above sea level. It is surrounded by the Gondar Zuria woredas. The total Livestock population of the study area is estimated to be 3.2 million cattle, 1.3 million sheep, 1.8 goats, 33, 000 horse, 13,000 mules and 400,000 donkeys (CSA, 2016).

\subsection{The Abattoir}

Gondar town has one municipal abattoir, one ELFORA abattoir and 98 legally registered butcheries that slaughter and supply the inspected meat to meat-sale shops of the town. Back yard slaughter is also significant in spite of some pressure from the government and it is common to see people consuming raw meat in butcheries. In the abattoir, on average 50 heads of cattle are slaughtered on non-fast days. Only one meat inspector conducts the anti-mortem and post mortem inspections. In the abattoir the number and species of animals slaughtered each day and the organs/carcass condemned with their respective causes were recorded. Carcasses and organs which are approved for consumption are delivered for meatsale shops of the town by vehicles.

\subsection{Study Population and Sample Size Determination}

A total of 500 animals were slaughtered at the abattoir between December, 2009 and April, 2010 and examined for tuberculous lesions. Of the 500 slaughtered animals, 468 were males and 32 were females that are culled because of old age or other reproductive problems. Cattle from Chelega, Debarke, Koladeba, Humera and Fogera sites of the country were the sources of animals for the abattoir. The age of each animal was determined based on dentition [18]. Body condition of each animal was estimated according Nicholson and Butterworth [19]. In the present study body condition is classified as lean, medium and fat.

To calculate the sample size an expected prevalence of $11 \%$ was taken on the basis of previous studies on the abattoir and using the Thrusfield formula [20] at 95\% confidence interval and 5\% desired absolute precision a total of 165 animals were required for the study. However, a total of 500 bovines were sampled.

\subsection{Study Design, Sample Collection and Transportation}

The study design for abattoir based survey was crosssectional study, which was conducted to determine the prevalence of BTB in the study area. The study was part of the Welcome Trust Funded Armauer Hansen Research Institute (AHRI) Bovine Tuberculosis project; hence, the study was designed to meet the overall objectives of the project in identifying and characterizing strains of $M$. bovis in the study site.

During necropsy procedures tissue lesions suspected to be positive for BTB were collected from lymph nodes of the head (retropharyngeal), lymph nodes associated with lungs (left bronchial, right bronchial, cranial mediastinal and caudal mediastinal) and from abdominal cavity lymph nodes (mesenteric lymph nodes) using universal bottles with phosphate buffer saline. Tissue samples collected from the abattoir were stored at $+4^{\circ} \mathrm{C}$ and transported to AHRI, ALERT TB laboratory for Mycobacterium culture using ice box packed with ice packs to maintain /keep the low temperature.

\subsection{Ante Mortem Inspection}

Animals were inspected for the presence of abnormalities and pulse rate, respiration rate and temperature were taken during ante mortem inspection. At Adama municipality abattoir there was no pre-slaughter tuberculin testing scheme. Animals which were unfit for slaughter were sent back and animals with sign of weakness and illness were delayed from being slaughtered for the subsequent day.

\subsection{Post Mortem Inspection}

Routine meat inspection procedures of carcass for the detection of visible abnormalities including tuberculous lesions were carried out by meat inspectors based on procedures adopted by the meat inspection and quarantine divisions of the Ministry of Agriculture of the country, Ethiopia [21]. The procedure involves examination of bronchial, mediastinal and pre-scapular lymph nodes and palpation and then incision of organs such as the lungs, liver and kidneys. Accordingly, the whole carcass is totally condemned if generalized suspected tuberculosis with the involvement of lymph nodes is detected, otherwise only affected organ or section of an organ is rejected [22].

In the detailed post mortem inspection procedures the following lymph nodes mandibular, medial, tracheobronchial, mediastinal, apical, retro-pharyngeal, parotid, sub-maxillary, mesenteric, supra-mammary and the lungs, liver, kidneys, spleen, mammary gland tissues, intestines, heart, thoracic and abdominal walls of selected carcasses were collected for a more thorough inspection. TB cases were defined based on gross characteristic lesions supplemented by the knowledge of pathogenesis and routes of lesion dissemination in the body [2]. The organs /tissues were cut in to multiple slices using separate scalpel blades the cut surfaces and internal portions were examined visually under bright light sources for the presence of TB lesions. 
Tissues with suspected TB lesions were collected separately in sterile universal bottles containing phosphate buffer saline solution and kept at $4{ }^{\circ} \mathrm{C}$ at the abattoir till it is transported to the AHRI TB laboratory center.

\subsection{Isolation of Mycobacteria}

On arrival at AHRI TB laboratory individual tissues were dissected and manually homogenized using a pestle and mortar followed by decontamination by shaking in an equal volume of a $4 \%$ sodium hydroxide $(\mathrm{NaOH})$ for 15 minutes and concentrated by centrifugation at 3000 revolutions per minute for 15 minutes. The sediment was neutralized with $2 \%$ hydrochloric acid $(\mathrm{HCl})$ using phenol red as indicator and then used to inoculate four different media slants of Lowenstein-Jensen (LJ) supplemented with $0.4 \%$ sodium pyruvate and glycerol (standard LJ) media with and without pyruvate and glycerol respectively. The slants were incubated at $37^{\circ} \mathrm{C}$ aerobically for six to eight weeks and examined on a weekly basis for the presences of Mycobacterial colonies. Cultures were considered negative if no visible growth was detected. Microscopic examination of cultures using the Ziehl-Neelsen staining method was performed to select Acid Fast Bacilli (AFB) positive isolates.

\subsection{Identification of Mycobacteria}

Identification of the Mycobacteria species was based on the rate of growth and colony morphology and growth on pyruvate or glycerol supplemented LJ media. Species belonging to the $M$. tuberculosis complex show a slow growth rate. For further identification of the Mycobacterial species, positive cultures were sub-cultured onto another set of LJ media supplemented with pyruvate or glycerol and incubated for another four weeks. Growth of M. bovis was enhanced by pyruvate whereas growth of $M$. tuberculosis is enhanced by glycerol. Mycobacteria were considered to be $M$. bovis if they grow slowly, showed small colony size and if their growth was enhanced by pyruvate [23].

\subsection{Data Analysis}

The data collected from the study area was entered in to an MS-Excel and then statistically analyzed using SPSS 17 version software. For all the analysis performed the confidence level was $95 \%$ and $p$ value less than or equal to 0.05 was set for significance. The statistical analyses used included comparison of proportions, and chi-square $\left(x^{2}\right)$ test
[20]. Test animal results were compared with different categories of variables such as sex, age, and breed, body condition of animals, post mortem result and culture result. Further post mortem was considered as a dependent variable and the effect of selected risk factors (age with three categories, sex with two categories, breed with two categories, body condition of an animal with three categories the range and frequency of anatomical sites with tuberculosis lesions) were recorded for each examined animal.

\section{Results}

\subsection{Abattoir Survey}

Animals passed for slaughter at the abattoir were examined for TB using the detailed meat inspection procedures and 43 animals were found with suspicious TB lesions by post mortem meat inspection and hence classified as tuberculous positive. Most common macroscopic lesions seen in affected tissue samples were the presence of circumscribed yellowish, calcified masses of various sizes and predominantly found in lymph nodes of the lung. Black demarcated necrotized areas were seen in some lymph nodes which contain active lesions.

\subsection{Distribution of Tuberculous Lesions in the Lymph Nodes}

The distribution of tuberculous lesions detected in post mortem examination of different lymph nodes of positive animals is shown in Table 1 bellow. The highest proportions of lesions were detected in the mediastinal lymph nodes $55.8 \%$ (24/43) followed by bronchial $27.9 \%(12 / 43)$ and $11.6 \%(5 / 43)$ was accounted by mesenteric lymph nodes and least proportion was accounted by the retropharyngeal lymph nodes $4.65 \%$ (2/43). Generally, $83.7 \%$ of lesions were detected in the thoracic cavity lymph nodes (mediastinal and bronchial) followed by mesenteric lymph nodes of the abdominal cavity and which accounted for $11.6 \%$ of the lesions and the remaining $4.65 \%$ were found in the lymph nodes of the head (retropharyngeal). During this study, TB lesions were not detected in the lungs, intestine, liver, kidneys, spleen and hepatic, submandibular and parotid lymph nodes and the carcasses with their associated lymph nodes. In this study anatomical site distributions of tubercle lesions were found to have statically significant value $(\mathrm{p}<0.05)$.

Table 1. Distribution of TB lesions in body lymph nodes.

\begin{tabular}{llll}
\hline Anatomical site & Frequency & Percentage & $\chi^{2}$ and P- value \\
\hline Apical (Bronchial) lymph nodes & 12 & $28 \%$ & \\
Mediastinal lymph nodes & 24 & $56 \%$ & $11.6 \%$ \\
Mesenteric lymph nodes & 5 & $2.6 \%$ & $289.8, P<0.05$ \\
Retropharyngeal lymph nodes & 2 & 2.05 \\
\hline
\end{tabular}

\subsection{Association of the Different Risk Factors with Prevalence of $B T B$}

The different host related and environmental risk factors were associated with the prevalence of BTB. Risk factors such as sex, body condition and origin were found to be the major risk factors and have showed statistically significant association with the occurrence of BTB. However, age and 
breed has insignificant association with the occurrence of BTB. The results are shown in Table 2 below.

Table 2. Association of the host related and environmental risk factors with the prevalence of $B T B$.

\begin{tabular}{|c|c|c|c|c|c|}
\hline Risk Factors & Number of animals examined & Positive for TB lesions & Percentage & $\chi^{2}$ & P-value \\
\hline \multicolumn{6}{|l|}{ Sex- } \\
\hline Male & 468 & 39 & $8.3 \%(39 / 468)$ & 147.179 & 0.025 \\
\hline Female & 32 & 4 & $12.5 \%(4 / 32)$ & & \\
\hline \multicolumn{6}{|l|}{ Breed- } \\
\hline Zebu & 489 & 43 & $8.8 \%(43 / 489)$ & 1.087 & 0.297 \\
\hline Cross & 11 & 0 & --- & & \\
\hline \multicolumn{6}{|l|}{ Age (years)- } \\
\hline $4-<7$ & 241 & 26 & $10.7 \%(26 / 241)$ & & 0.187 \\
\hline$>7$ & 233 & 27 & $11.6 \%(27 / 233)$ & & \\
\hline \multicolumn{6}{|c|}{ Body condition- } \\
\hline & Lean-32 & 9 & $28.1 \%(9 / 32)$ & 33.57 & 0.000 \\
\hline & Med-252 & 33 & $13.1 \%(33 / 252)$ & & \\
\hline & Fat-216 & 11 & $5.1 \%(11 / 216)$ & & \\
\hline \multicolumn{6}{|l|}{ Origin } \\
\hline Chelega & 92 & 11 & $12 \%(11 / 92)$ & & \\
\hline Koladeba & 82 & 6 & $7.3 \%(6 / 82)$ & & \\
\hline Fogera & 115 & 7 & $6 \%(7 / 115)$ & 23.688 & 0.011 \\
\hline Debarke & 98 & 10 & $10.2 \%(10 / 98)$ & & \\
\hline Humera & 113 & 9 & $8 \%(9 / 113)$ & & \\
\hline
\end{tabular}

Table 2 above shows that the risk factors sex $\left(\chi^{2}=147.179\right.$, $\mathrm{P}$ value 0.025$)$, body condition $\left(\chi^{2}=17.34, \mathrm{P}<0.05\right)$ and origin $\left(\chi^{2}=23.688, \mathrm{p}\right.$ value 0.011$)$ have a significant association with the occurrence of BTB, in that females $(17.7 \%)$ recorded the highest prevalence than males $(9.8 \%)$ and lean animals rates highest (28.1\%) and fat ones being the least (5.1\%). Bovines of origin Chelega have also recorded the highest prevalence $(12 \%)$ and that of Fogera being the least $(4.95 \%)$. However, age $\left(\chi^{2}=3.357, \mathrm{P}\right.$ value 0.187$)$ and breed $\left(\chi^{2}=1.087, \mathrm{P}\right.$ value, 0.297), has shown insignificant association with the occurrence of BTB.

\subsection{Isolation of Mycobacteria}

Out of the 43 samples which were suspected for tuberculosis and sent to AHRI (Harmeuar Hansen Research institute) TB laboratory center and cultured on LJ media, 5 has shown colony growths on LJ media and positive for AFB test up on Ziehl-Neelsen staining technique.

\subsection{Identification of Mycobacteria}

Identification of the Mycobacteria species were based on the rate of growth and colony morphology and growth on pyruvate or glycerol enriched media. Among the 5 cultures and AFB positive samples 3 samples were grown on pyruvate enriched media and 2 were grown on glycerol enriched media. Dysgonic growths after eight weeks were found on media enriched with pyruvate and colonies were moist, round and whitish. Whereas eugonic growths were observed after 2-3 weeks on the media enriched with glycerol.

\section{Discussion}

Bovine Tuberculosis is caused by intercellular infection with acid fast bacterium, M. bovis. Bovine exposure to these organisms can result in a chronic disease that jeopardizes animal welfare and productivity in some countries leading to significant economic loses. Moreover, human TB of animal origin caused by $M$. bovis is becoming important in developing countries. In Ethiopia, although the endemic nature of BTB has been known, several works were done in different areas of the country.

In the present study $8.6 \%$ prevalence of BTB was recorded based on post mortem examination in the study area, Gondar ELFORA abattoir. The same studies were also undertaken in different parts of the country of which a study carried in Gondar abattoir by Habtom [24] recorded 11.6\%.

The same study was also conducted in Adama municipality abattoir with a prevalence rate of $5.2 \%$ by Ameni and Wudie [25] and $12.1 \%$ by Anebo [26], in Hossana 4.5\% [14] and in Addis Ababa and Debre zeit abattoirs [27] have recorded a prevalence of $0.8 \%$ of the 144,487 slaughtered animals and an overall prevalence report of $0.052 \%$ were stated from a total of $1,336,266$ slaughtered cattle in eight export abattoirs in the year 1999-2005. The infection rate in cattle has been found to differ greatly from place to place [27], and this difference is most likely linked to the type of the production system followed.

However, the findings of the present study is lower when compared to the previous reports carried in Wolayta Sodo abattoir 14.8\% [10], in Jinka 15.4\% [28] and 14.8\% [29], in Gondar abattoir 15.4\% [30], in Butajira municipality abattoir $20.96 \%$ [31] and in Deber zeit dairy farms 50\% [10]. The lower prevalence rate recorded in the study area might be due to the difference in the sample size considered by different studies or basically due to the difference in origin of the slaughtered animals.

Necropsy inspection results of the study abattoir revealed the detection of only $8.6 \%$ (43/500) visible lesions detected of the carcasses inspected. In the abattoir, smaller lesions could possibly be missed due to limited time available for the inspection of each tissue. Besides, the sensitivity of gross 
post mortem examination is also affected by the method employed and the anatomical site examined which were generally poor and need further improvement and this was an idea also stated by Teklu et al. [14].

In the present study $83.7 \%$ of the tuberculous lesions most frequently and intensively were found in the thoracic cavity lymph nodes, followed by $11.6 \%$ in the abdominal cavity (mesenteric lymph nodes) and the remaining $4.65 \%$ in the lymph nodes of the head (retropharyngeal lymph nodes). This finding is comparable with the previous results indicated in Gondar ELFORA abattoir [24], Jinka municipality abattoir [28] and Butajira municipality abattoir [31]. This finding is also similar to those previous reports of Oloya et al. [32].

These agreeable results likely indicate that most of tuberculosis infection is acquired by aerosol route. However, this finding is in contrast with previous results of Terefe [30] indicating a higher prevalence of TB lesions in the abdominal cavity lymph nodes (mesenteric lymph nodes) which shows that infection is through ingestion route and this result is also supported by Radostits and Blood [33]. The present as well as previous results imply that both thoracic and abdominal cavity lymph nodes could possibly be involved and thorough focus should be given during post mortem examinations to both cavity lymph nodes.

In the present study 5 out of the 43 suspicious tissue samples, $11.6 \%$ (5/ 43), were grown on LJ pyruvate enriched media and glycerol media. This result is higher than the previous results conducted in Gondar ELFORA abattoir [24] $8.5 \%$ and Woldiya $6 \%$ [34] respectively But was found to be lower than the previous results conducted in Adama municipality abattoir $27.7 \%$ [26], Jinka $15.4 \%$ and $19.1 \%$ $[28,29]$ in Gondar abattoir 25.95\% [30] and in Butajira $13.51 \%$ [35], This difference could be due to either losses of the agent during freezing or due to delayed transportation from the site of collection [6]. Indicated losses of 5-10\% due to contamination resulting from prolonged preservation and loses up to $60 \%$ due to decontamination procedures are also reported. The use of proper time in culturing and application of standard laboratory technique could possibly increase the chance of recovery in AFB tests.

The other finding of this study indicates higher isolation of Mycobacterium from thoracic cavity lymph nodes; mediastinal lymph nodes, bronchial lymph node and least amount of Mycobacterium were isolated from mesenteric and retropharyngeal lymph nodes respectively. This finding is in agreement with the previous reports, in Butajira [31], in Gondar abattoir [24], and in Jinka [28]; this suggests that mediastinal lymph nodes are the most probable affected lymph node over the other. However, in contrast with previous report in Gondar abattoir [30], indicated higher isolation Mycobacterium from mesenteric lymph nodes.

The higher prevalence of BTB of the present study was recorded in animals under poor body condition score, followed by animals with medium body condition and then animals under good body condition score. This finding do strongly agree with many of the previous reports in different corner of the country, in Adama [25], in Gondar [24, 30], in Hosanna [14], in Jinka [28, 29], and in Butajira [31, 35]. This may indicate that the animals under good body condition are with good immune status that can respond to any foreign invading agent better than those with poor body condition which can be immune compromised due to other diseases and malnourished as malnutrition and concurrent infections depress the immunity of the animal, in some cases so that TB lesions develop [36].

In this study absence of significant differences in infection rates based on age and breed is contraindicated with previous reports [14, 37]. This indicates the presence of other factors that may play a significant role in the spread of tuberculosis in this environment. Possible risk factors include management (confinement), herd size and location, in relation to proximity to already infected farms [38]. A relatively higher proportion of females were infected, possibly due to their longer productive life and other stressful factors (such as pregnancy, parturition, lactation, etc.) associated with female animals [39, 40 \& 41]. Cattle originated from Chelega showed relatively higher rate of infection and cattle originated from fogera had lower rate of infection.

\section{Conclusions}

Bovine Tuberculosis is a zoonotic disease caused by intercellular infection with an acid fast bacterium, Mycobacterium bovis. In this study, a relatively high variation in the prevalence of BTB existed among cattle originating from different origins, body condition scores and sex. High proportion of tubercles were found in the thoracic cavity lymph nodes, followed by abdominal cavity lymph nodes and the least proportion accounted by lymph nodes of the head. Identification and characterization of the Mycobacterial species was done based on the rate of growths, colony morphology and AFB tests, long delay during transportation and storage can cause loses of AFB positive samples. Risk factors such as sex and body condition had significant association with post mortem result whereas age and breed did not have association with post mortem results. At the abattoir there is no pre slaughter tuberculin test and smaller lesions could be missed due to limited time available for the examination of each tissue. In addition to this, meat inspection is not conducted to assess TB and the inspector did not give emphasis to thoracic and abdominal cavity lymph nodes up on which the higher proportions of tubercle lesions are found. Consumption of raw meat is also a common habit in the town.

Therefore, based on the above conclusion the following recommendations are forwarded:

a. The abattoir inspection procedures should be improved as the sensitivity of gross postmortem examination is affected by the method employed and the anatomical site examined.

b. Public awareness on the transmission of BTB should be given and consumption of cooked meat should be 
encouraged too.

c. During slaughter, post mortem examination should focus on thoracic and abdominal cavity lymph nodes as these are the sites where the higher proportion of tubercle lesions found.

d. The use of proper time in culturing and application of standard laboratory techniques could possibly increase the chance of recovery in AFB tests

\section{Competing Interests}

As an author we are certifying that there is no conflict of interest regarding the publication of this paper.

\section{Acknowledgements}

I would like to say thank you for Mekelle University and AHRI, ALERT campus for their financial support to accomplish the thesis work

\section{References}

[1] OIE, 2009. Office International des Epizooties (OIE) Terrestrial manual: Bovine Tuberculosis, World Health Organization for Animal Health, Paris. Chapter 2, 4, 7.

[2] Pollock, J. M., Welsh, M. D. and Mc Nair, J. 2005. Immune Responses In Bovine Tuberculosis towards the New Strategies for the Diagnosis and Control of Disease Veterinary Immune and Immunopathology, 108: 37-43.

[3] Ashioford, D. A., Whitney, E., Rahunathan, P. and Cosivi, A. 2001. Epidemiology of Selected Mycobacteria That Infect Human and Other Animals Technical and Scientific Review, Office of International des Epizootics., 20: 105-112.

[4] Biet, F., Boschiroli, M. L., Thorel, M. F. and Guilloteau, L. A., 2005. Zoonotic Aspects of M. bovis And M. aviumIntracellular Complex. Veterinary Research, 36.

[5] Kazwala, R. R., Kambarage, D. M., Daborn, C. J., Nyange, J., Jiwa, S. F. and Sharp, J. M. (2001). Risk factors associated with the occurrence of bovine tuberculosis in cattle in the Southern Highlands of Tanzania. Veterinary Research Communication, 25: 609-614.

[6] WHO (2009). Global tuberculosis control: Epidemiology, strategy, financing. World Health Organization. Geneva, Switzerland.

[7] Cockele, J. W. and Tustin, R. C., 2004. Infectious Diseases of Livestock $2^{\text {nd }}$ ed Volume. 3. Oxford University Press Southern Africa Pp 1973-1990.

[8] AMENI, G. and ROGER, F. 1998. Study on the epidemiology of bovine tuberculosis in dairy farms (Debre Zeit and Ziway, Ethiopia). In: Proceeding of the 12th Conference of the Ethiopian Veterinary Association (EVA), Addis Ababa, Ethiopia, 13-19.

[9] KIROS, T. 1998. Epidemiology and zoonotic importance of bovine tuberculosis in selected sites of Eastern Shoa, Ethiopia. Faculties of Veterinary Medicines of Addis Ababa University an Free University of Berlin, MSc Thesis.
[10] Ameni, G., Regassa, A., Kassa, I., and Medhin, G., 2001. Survey on Bovine Tuberculosis and Its Public Health Implication to Cattle raising Families In Wolaita Sodo, Southern Ethiopia. Journal Animal Production, 1: Pp. 5562 .

[11] Bogale, A., Lubke-Beker, A., Lemma, E., Kiros, T., Britton, S. 2001. Bovine Tuberculosis: A Cross Sectional and Epidemiological Study In and Around Addis Ababa. Bulletin of Animal Health Production in Africa, 48: 71-80.

[12] Corner, L. A., Melville, L., M., Cubbin, K., Small, K. J., Mc Corminen, B. S., Wood, P. R and Rothel, J. S., 1994. Efficiency of Inspection Procedure for The Detection of Tuberculosis Lesions in Cattle, 67 (11): 381-392.

[13] Whipple D. L., Bolin C. A., Miller J. M. (1996): Distribution of lesions in cattle infected with $M$. bovis. Journal of Veterinary Diagnostic and Investment, 8: 351-354.

[14] Teklu, A., Asseged, B., Yimer, E., Gebeyehu, M. and Woledesenbet, Z. 2004. Tuberculosis Lesions do not detected by Routine Abattoir Inspection. The Experience of the Hossana Municipality Abattoir Southern Ethiopia. Rev Science Technology, 23(3): 957-964.

[15] Thoen C. O., Huchzermeyer H., Himes E. M. (1995): Laboratory diagnosis of bovine tuberculosis. In: Thoen C. O., Steele J. H. (eds.): M. bovis Infection in Animals and Humans. Iowa State University Press, Ames, Iowa, USA. 6372.

[16] Wards B. J., Collins D. M., de Lisle G. W. (1995): Detection of Mycobacterium bovis in tissues by polymerase chain reaction. Journal of Veterinary Microbiology, 43: 227-240.

[17] Demelash, B., Inangolet, F., Oloya, J., Asseged, B., Badaso, M., Yilkal, E and Skjerve, E. 2008. Prevalence of Bovine Tuberculosis in Ethiopia Slaughter Cattle Based on Post Mortem Examination.

[18] Cosivi O, Grange JM, Daborn CJ, Raviglione MC, Fujikura T, 1998. Zoonotic tuberculosis due to Mycobacterium bovis in developingountries. Emerging Infectious Disease, 4(1): 1-17.

[19] Nicholson MJ, Butterworth MH (1986). A guide to condition scoring of zebu cattle. ILRI/FAO, Addis Ababa, Ethiopia.

[20] Thrusfield, M., 2005. Veterinary Epidemiology. 3rd ed. Blackwell science ltd, Oxford,

[21] Hailemariam S. (1975): A brief analysis of activities of Meat Inspection and Quarantine Division, Department of Veterinary Service, Ministry of Agriculture, Addis Ababa, Ethiopia.

[22] Asseged, B., Woledesenbet, Z., Yimer, E. and Lemma, E. 2004. Evaluation of Abattoir Inspection for the Diagnosis of Mycobacterium bovis Infection in Cattle at Addis Ababa Abattoir. Tropical Animal Health production, 36 (6): 537-546.

[23] de Kantor, I. N., Nadar, A., Bernarden, A., Grano, O. D and Man, E. 1998. Tuberculosis in Cattle not Detected By Slaughter House Inspection. Journal of Veterinary Medicine.

[24] Habtom, A. 2008. Cross a Sectional Study of Bovine TB in Gondar ELFORA Abattoir, North Gondar, Ethiopia. DVM, Thesis, Faculty of Veterinary Medicine, Addis Ababa University, Deberizeit, Ethiopia.

[25] Ameni, G. and Wudie, A., 2003. Preliminary Study on Bovine Tuberculosis In Adama. 
[26] Anebo, M., 2007. Prevalence of Bovine Tuberculosis in Adama Municipality Abattoir DVM Thesis. Faculty of Veterinary Medicine, Addis Ababa University, Debre zeit, Ethiopia.

[27] Shitaye, J. E., Tsegaye, W. and Pavlik, I. 2007. Bovine Tuberculosis Infection in Animal and Human Population in Ethiopia: A review Veterinary Medicine Pp. 317-332.

[28] Taddele, H. 2006. A Cross Study of Bovine Tuberculosis in Jinka Municipal Abattoir, Southern Ethiopia DVM Thesis. Faculty of Veterinary Medicine, Addis Ababa University, Debre zeit, Ethiopia.

[29] Degene, Y.2007. A Cross Sectional Study Of Bovine TB In Jinka Municipal Abattoir Southern Ethiopia, DVM, Thesis, Faculty Of Veterinary Medicine, Addis Ababa University, Deberizeit, Ethopia.

[30] Terefe, Y. 2007. A Cross-sectional study of Bovine TB: In Gondar ELFORA Abattoir. DVM Thesis, Faculty of Veterinary Medicine, Addis Ababa University, Debre zeit, Ethiopia.

[31] Elias, K., Hussein, D. Asseged, B. Wondwossen, T, T. and Gebeyehu, M. (2008). Bovine tuberculosis in Addis Ababa dairy farms. Review of Science and Technology, Office International des Epizooties, 27: 915-923.

[32] Oloya J, Kazwala R, Lund A, Opuda-Asibo J, Demelash B. 2007 Characterization of Mycobacteria isolated from slaughter cattle in pastoral regions of Uganda. BMC Microbiology, 7: 95.

[33] Radostits OM, Blood DC (1994). Disease caused by mycobacteria IV. In: Veterinary Medicine. 7th Ed. UK: Bailliere Tindall, London, pp. 710-740.

[34] Hassen, A. 2006. A Cross Sectional Study of Bovine Tuberculosis In Wolidya Municipal Abattoir North Wollo, Ethiopia, DVM Thesis, Faculty of Veterinary Medicine, Addis Ababa University, Debre zeit, Ethiopia.
[35] Negesse, W. 2007. Cross Sectional Study of Bovine Tuberculosis in Butajira Municipality Abattoir DVM, thesis, Faculty of veterinary medicine, Addis Ababa University, Debre zeit, Ethiopia.

[36] Collins FM (1994). The immune response to mycobacterial infection: development of new vaccines Veterinary Microbiology, 40: 95-110.

[37] Ayele, W. Y., Neill, S. D., Zinsstag, J., Weiss, M. G. and Pavlik, I. 2004. Bovine tuberculosis: an old disease but a new threat to Africa. International Journal of Tubercle and Lung Disease, 8:924-937.

[38] Bogale A, Liibke-becker A, Lemma E, Kirose T, Britton S. 2000. Bovine tuberculosis: A cross-sectional and Epidemiological study in and around Addis Ababa. Bulletin of Animal Health Production in Africa 48:71-80

[39] Asseged B, Lübke-Becker A, Lemma E, Taddele K, Britton S (2000). Bovine tuberculosis: a cross-sectional and epidemiological study in and around Addis Ababa. Bulletin of Animal Health Production in Africa, 48:71-80.

[40] Müller B, Steiner B, Bonfoh B, Fane A, Smith NH, 2008. Molecular characterisation of Mycobacterium bovis isolated from cattle slaughtered at the Bamako abattoir in Mali. BMC Veterinary Research, 4: 26.

[41] Radostits O. M., Gray G. C., Blood D. C., Hinchelift K. W. (2000). A text Book of Disease of Cattle, Sheep, Pig, Goat and Horses. In: Veterinary Medicine. 9th ed. Harcourt Publisher Ltd., London. 909-918.

[42] Central Statistical Agency (CSA) 2016. Federal democratic republic of Ethiopia. Central statistical agency. Agricultural sample survey, Volume II, Report on livestock and livestock characteristics. Statistical bulletin 583, Addis Ababa, Ethiopia. 